\title{
MODERN DEVELOPMENTS IN FLOW CONTROL
}

Mohamed Gad-el-Hak

Department of Aerospace \& Mechanical Engineering

University of Notre Dame

Notre Dame, IN 46556

Appeared in Applied Mechanics Reviews, vol. 49, pp. 365-379, 1996. 


\title{
MODERN DEVELOPMENTS IN FLOW CONTROL
}

\author{
Mohamed Gad-el-Hak \\ Department of Aerospace \& Mechanical Engineering \\ University of Notre Dame \\ Notre Dame, IN 46556
}

\begin{abstract}
This brief article reviews the important advances in the field of flow control that took place during the past few years. This broad area of research remains of great interest for its numerous potential benefits for both the military and civilian sectors. Spurred by the recent developments in chaos control, microfabrication and neural networks, reactive control of turbulent flows is now in the realm of the possible for future practical devices. Other less complex control schemes, passive as well as active, are more market ready and are also witnessing resurgence of interest.
\end{abstract}

\section{INTRODUCTION}

The ability to manipulate a flow field to effect a desired change is of immense practical importance. As a scientific discipline and as a technological curiosity, flow control is perhaps more hotly pursued by scientists and engineers than any other area in fluid mechanics. The present author reviewed the subject seven years ago (Gad-el-Hak, 1989), and was invited by the Editor-inChief of the Applied Mechanics Reviews to update that article. Other reviews that appeared since 1989 include those by Bushnell and McGinley (1989), Fiedler and Fernholz (1990), Gad-el-Hak and Bushnell (1991), Moin and Bewley (1994), and Gad-el-Hak (1994). Several useful articles are contained within the book edited by Bushnell and Hefner (1990).

\subsection{Five Eras of Flow Control}

Flow control involves passive or active devices to effect a beneficial change in wallbounded or free-shear flows. Whether the task is to delay/advance transition, to suppress/enhance turbulence or to prevent/provoke separation, useful end results include drag reduction, lift enhancement, mixing augmentation and flow-induced noise suppression. Broadly, there are 
perhaps five distinct eras in the development of the art and science of this challenging albeit very useful field of research and technology: The empirical era (prior to 1900); the scientific era (19001940); the World War II era (1940-1970); the energy crisis era (1970-1990); and the 1990s and beyond. The art of flow control probably has its roots in prehistoric times when streamlined spears, sickle-shaped boomerangs, and fin-stabilized arrows evolved empirically by archaic Homo sapiens. Relatively soon after the dawn of civilization and the establishment of an agriculture way of life 8,000 years ago, complex systems of irrigation were built along inhabited river valleys to control the water flow, thus freeing man from the vagaries of the weather. For centuries, farmers knew the value of windbreaks to keep top soil in place and to protect fragile crops.

The science of flow control originated with Prandtl (1904), who, in a mere 8-page manuscript, introduced the boundary layer theory, explained the physics of the separation phenomena and described several experiments in which a boundary layer was controlled. Thus the birth of the scientific method to control a flow field. Slowly but surely, the choice of flow control devices is no longer a trial and error feat, but physical reasoning and even first principles are more often than not used for rational design of such artifacts.

Stimulated by the Second World War and the subsequent cold war, that trend accelerated significantly during the third era (1940-1970). Military needs of the superpowers dictated the development of fast, highly maneuverable, efficient aircraft, missiles, ships, submarines and torpedoes, and flow control played a major role in achieving these goals. Natural laminar flow, laminar flow control and polymer drag-reduction are notable achievements during this era. Partial summaries of flow control research during this period are contained within the books edited by Lachmann (1961) and Wells (1969).

The energy crises exemplified by the 1973 Arab oil embargo brought about a noticeable shift of interest from the military sector to the civilian one. During the period 1970-1990, government agencies and private corporations around the world but particularly in the industrialized countries invested valuable resources searching for methods to conserve energy, and hence drag reduction for civilian air, sea and land vehicles, for pipelines and for other industrial 
devices was emphasized. The availability of fast, inexpensive computers made it possible to simulate numerically complex flow situations that have not been approachable analytically. Some control strategies, for example transition-delaying compliant coatings (Gad-el-Hak, 1996), were rationally optimized using computational fluid dynamics. Large-eddy breakup devices (LEBUs) and riblets are examples of control methods developed during this period to reduce skin-friction drag in turbulent boundary layers. Good sources of information on these and other devices introduced during the fourth era are the books edited by Hough (1980), Bushnell and Hefner (1990), and Barnwell and Hussaini (1992). Numerous meetings devoted to flow control, particularly drag reduction, were held during this period. Plentiful fuel supplies during the 1990s and the typical short memory of the long gas lines during 1973 have, unfortunately, somewhat dulled the urgency and enthusiasm for energy conservation research as well as practice.

For the 1990s and beyond, more complex reactive control devices, geared specifically towards manipulating the omnipresent coherent structures in transitional and turbulent shear flows (Cantwell, 1981; Robinson, 1991), are pursued by several researchers. Theoretical advances in chaos control and developments of microelectromechanical systems (MEMS) and neural networks should help such efforts. Papers specifically addressing reactive control strategies include those by Wilkinson (1990), Moin and Bewley (1994), and Gad-el-Hak (1994).

\subsection{The Taming of the Shrew}

Considering the extreme complexity of the turbulence problem in general and the unattainability of first-principles analytical solutions in particular, it is not surprising that controlling a turbulent flow remains a challenging task, mired in empiricism and unfulfilled promises and aspirations. Brute force suppression, or taming, of turbulence via active, energyconsuming control strategies is always possible, but the penalty for doing so often exceeds any potential savings. The artifice is to achieve a desired effect with minimum energy expenditure. Indeed, suppressing turbulence is as arduous as the taming of the shrew. The former task will be emphasized throughout this presentation, but for now we reflect on a short verse from the latter. 
From William Shakespeare's The Taming of the Shrew:

Curtis (Petruchio's servant, in charge of his country house): Is she so hot a shrew as she's reported?

Grumio (Petruchio's personal lackey): She was, good Curtis, before this frost. But thou know'st winter tames man, woman, and beast; for it hath tamed my old master, and my new mistress, and myself, fellow Curtis.

\subsection{Outline}

The present article reviews the important developments in the field of flow control that took place during the past seven years and suggests avenues for future research. The emphasis will be on reactive flow control for future vehicles and other industrial devices, but more market-ready methods of control will be briefly mentioned. Because of the limited space apportioned, this article will not detail any of the recent achievements in the field but will mostly serve as a navigation tool to access the available literature. The number of existing articles and books on the subject is daunting. For example, a recent bibliography on the relatively narrow sub-subject of skin-friction reduction by polymers and other additives cites over 4,900 references (Nadolink and Haigh, 1995). Therefore, citations in the present short article will be rather selective, indeed very limited.

Following these introductory remarks, a brief description of the unifying principles of flow control is given in Section 2. Sections 3 contrasts wall-bounded flows to free-shear flows, and includes more traditional approaches to flow control. In Section 4, recent developments in the area of reactive flow control are discussed. Sections 5, 6 and 7 consider the emerging areas of chaos control, microfabrication and neural networks, respectively, as they relate to reactive control strategies. Finally, brief concluding remarks are given in Section 8.

\section{UNIFYING PRINCIPLES}

Section 3 will contrast free-shear and wall-bounded flows. For the purposes of the present section, we focus on the latter particularly the technologically very important boundary layers. An external wall-bounded flow, such as that developing on the exterior surface of an aircraft or a 
submarine, can be manipulated to achieve transition delay, separation postponement, lift increase,

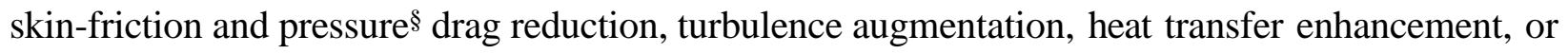
noise suppression. These objectives are not necessarily mutually exclusive. For example, by maintaining as much of a boundary layer in the laminar state as possible, the skin-friction drag and the flow-induced noise are reduced. However, a turbulent boundary layer is in general more resistant to separation than a laminar one. By preventing separation, lift is enhanced and form drag is reduced. Moreover, mixing and heat transfer are enhanced by the turbulence. The challenge in choosing a flow control scheme is of course to achieve a beneficial goal at a minimum cost, without adversely affecting another goal. An ideal method of control that is simple, is inexpensive to build and to operate, and does not have any trade-off does not exist, and the skilled engineer has to make continuous compromises to achieve a particular design objective.

There are different classification schemes for flow control methods. One is to consider whether the technique is applied at the wall or away from it. Surface parameters that can influence the flow include roughness, shape, curvature, rigid-wall motion, compliance, temperature, and porosity. Heating and cooling of the surface can influence the flow via the resulting viscosity and density gradients. Mass transfer can take place through a porous wall or a wall with slots. Suction and injection of primary fluid can have significant effects on the flow field, influencing particularly the shape of the velocity profile near the wall and thus the boundary layer susceptibility to transition and separation. Different additives, such as polymers, surfactants, micro-bubbles, droplets, particles, dust or fibers, can also be injected through the surface in water or air wallbounded flows. Control devices located away from the surface can also be beneficial. Large-eddy breakup devices (also called outer-layer devices, or OLDs), acoustic waves bombarding a shear layer from outside, additives introduced in the middle of a shear layer, manipulation of freestream turbulence levels and spectra, gust, and magneto- and electro-hydrodynamic body forces are examples of flow control strategies applied away from the wall.

\footnotetext{
$\S$ Pressure drag includes contributions from flow separation, displacement effects, induced drag, wave drag, and, for time-dependent flows, virtual mass.
} 
A second scheme for classifying flow control methods considers energy expenditure and the control loop involved. As shown in the schematic in Figure 1, a control device can be passive, requiring no auxiliary power, or active, requiring energy expenditure. As for the action of passive devices, some prefer to use the term flow management rather than flow control (Fiedler and Fernholz, 1990), reserving the latter terminology for dynamic processes. Active control is further divided into predetermined or reactive. Predetermined control includes the application of steady or unsteady energy input without regard to the particular state of the flow. The control loop in this case is open as shown in Figure 2a, and no sensors are required. Reactive ${ }^{\S}$ control is a special class of active control where the control input is continuously adjusted based on measurements of some kind. The control loop in this case can either be an open, feedforward one (Figure 2b) or a closed, feedback loop (Figure 2c). Classical control theory deals, for the most part, with reactive control.

The distinction between feedforward and feedback is particularly important when dealing with the control of flow structures which convect over stationary sensors and actuators. In feedforward control, the measured variable and the controlled variable differ. For example, the pressure or velocity can be sensed at an upstream location, and the resulting signal is used together with an appropriate control law to trigger an actuator which in turn influences the velocity at a downstream position. Feedback control, on the other hand, necessitates that the controlled variable be measured, fed back and compared with a reference input. Reactive feedback control is further classified into four categories: Adaptive, physical model-based, dynamical systems-based, and optimal control (Moin and Bewley, 1994). We will return to these categories in Section 4.2.

A yet another classification scheme is to consider whether the control technique directly modifies the shape of the instantaneous/mean velocity profile or selectively influence the small dissipative eddies. An inspection of the Navier-Stokes equations written at the surface (see, for example, Gad-el-Hak, 1990), indicates that the spanwise and streamwise ${ }^{\S \S}$ vorticity fluxes at the

\footnotetext{
$\S$ Also termed by some interactive flow control. Reactive could be confused with chemically reacting flows, but is more proper linguistically to describe this particular control strategy.

$\S \S$ Streamwise vorticity exists only if the velocity field is three-dimensional, instantaneously or in the mean.
} 
wall can be changed, either instantaneously or in the mean, via wall motion/compliance, curvature, suction/injection, streamwise or spanwise pressure-gradient (respectively), or normal viscositygradient. These vorticity fluxes determine the fullness of the corresponding velocity profiles. For example, suction, favorable pressure-gradient or lower wall-viscosity results in vorticity flux away from the wall, making the surface a source of spanwise and streamwise vorticity. The corresponding fuller velocity profiles have negative curvature at the wall and are more resistant to transition and to separation but are associated with higher skin-friction drag. Conversely, an inflectional velocity profile can be produced by injection, adverse pressure-gradient or higher wallviscosity. Such profile is more susceptible to transition and to separation and is associated with lower, even negative, skin friction. Note that many techniques are available to effect a wall viscosity-gradient; for example surface heating/cooling, film boiling, cavitation, sublimation, chemical reaction, wall injection of lower/higher viscosity fluid, and the presence of shear thinning/thickening additive.

Flow control devices can alternatively target certain scales of motion rather than globally changing the velocity profile. Polymers, riblets and LEBUs, for example, appear to selectively damp only the small dissipative eddies in turbulent wall-bounded flows. These eddies are responsible for the (instantaneous) inflectional profile and the secondary instability in the buffer zone, and their suppression leads to increased scales, a delay in the reduction of the velocity-profile slope, and consequent thickening of the wall region. In the buffer zone, the scales of the dissipative and energy containing eddies are roughly the same and, hence, the energy containing eddies will also be suppressed resulting in reduced Reynolds stress production, momentum transport and skin friction.

\section{WALL-BOUNDED AND FREE-SHEAR FLOWS}

A particular control strategy is chosen based on the kind of flow and the control goal to be achieved. Presence or lack of walls, Reynolds number, Mach number, and the character of the flow instabilities are all important considerations for the type of flow to be controlled. These 
issues are discussed in turn in the present section. Section 3 will conclude with a brief listing of traditional and market-ready control strategies, reserving a discussion of futuristic reactive control systems to the following section.

\subsection{Inviscid and Viscous Instabilities}

Free-shear flows, such as jets, wakes or mixing layers, are characterized by inflectional mean-velocity profiles and are therefore susceptible to inviscid instabilities. Viscosity is only a damping influence in this case, and the prime instability mechanism is vortical induction. Control goals for such flows include transition delay/advancement, mixing enhancement and noise suppression. External and internal wall-bounded flows, such as boundary layers and channel flows, can too have inflectional velocity profiles, but, in the absence of adverse pressure-gradient and similar effects, are characterized by non-inflectional profiles and viscous instabilities are then to be considered. This kind of viscosity-dominated wall-bounded flows are intrinsically stable and therefore are generally more difficult to control. Free-shear flows and separated boundary layers, on the other hand, are intrinsically unstable and lend themselves more readily to manipulation.

Free-shear flows originate from some kind of surface upstream be it a nozzle, a moving body or a splitter plate, and flow control devices can therefore be placed on the corresponding walls albeit far from the fully-developed regions. Examples of such control include changing of the geometry of a jet exit from circular to elliptic (Gutmark and Ho, 1986), using periodic suction/injection in the lee side of a blunt body to affect its wake (Williams and Amato, 1989), and vibrating the splitter plate of a mixing layer (Fiedler et al., 1988). These and other techniques are

extensively reviewed by Fiedler and Fernholz (1990), who offer a comprehensive list of appropriate references, and more recently by Gutmark et al. (1995) and Viswanath (1995).

\subsection{Regimes of Reynolds and Mach Numbers}

Reynolds number determines whether the flow is laminar or turbulent. For low-tomoderate Reynolds numbers, the flow is laminar. Because of the nature of their instabilities, freeshear flows undergo transition at extremely low Reynolds numbers as compared to wall-bounded flows. Many techniques are available to delay laminar-to-turbulence transition for both kinds of 
flows, but none would do that to indefinitely high Reynolds numbers. Therefore, for Reynolds numbers beyond a reasonable limit, one should not attempt to prevent transition but rather deal with the ensuing turbulence. Of course early transition to turbulence can be advantageous in some circumstances, for example to achieve separation delay, enhanced mixing or augmented heat transfer. The task of advancing transition is generally simpler than trying to delay it.

Three Reynolds number regimes can be identified for the purpose of reducing skin friction in wall-bounded flows. First, if the flow is laminar, typically at Reynolds numbers based on distance from leading edge $<10^{6}$, then methods of reducing the laminar shear stress are sought. These are usually velocity-profile modifiers, for example adverse-pressure gradient, injection, cooling (in water) and heating (in air), that reduce the fullness of the profile at the increased risk of premature transition and separation. Secondly, in the range of Reynolds numbers from $1 \times 10^{6}$ to $4 \times 10^{7}$, active and passive methods to delay transition as far back as possible are sought. These techniques can result in substantial savings and are broadly classified into two categories: stability modifiers and wave cancellation (Gad-el-Hak, 1989). The skin-friction coefficient in the laminar flat-plate can be as much as an order of magnitude less than that in the turbulent case. Note, however, that all the stability modifiers, such as favorable pressure-gradient, suction or heating (in liquids), result in an increase in the skin friction over the unmodified Blasius layer. The object is, of course, to keep this penalty below the potential saving; i.e., the net drag will be above that of the flat-plate laminar boundary-layer but presumably well below the viscous drag in the flat-plate turbulent flow. Thirdly, for $R e>4 \times 10^{7}$, transition to turbulence cannot be delayed with any known practical method without incurring a penalty that exceeds the saving. The task is then to reduce the skin-friction coefficient in a turbulent boundary layer. Relaminarization (Narasimha and Sreenivasan, 1979) is an option, although achieving a net saving here is problematic at present.

Mach number determines whether the flow is incompressible $(M a<0.3)$ or compressible $(M a>0.3)$. The latter regime is further divided into subsonic $(M a<1)$, transonic $(0.8<M a<1.2)$, supersonic $(M a>1)$, and hypersonic $(M a>5)$. Each of those flow regimes lends itself to different optimum methods of control to achieve a given goal. Take laminar-to- 
turbulence transition control as an illustration (Bushnell, 1994). During transition, the field of initial disturbances is internalized via a process termed receptivity and the disturbances are subsequently amplified by various linear and nonlinear mechanisms. Assuming that by-pass mechanisms, such as roughness or high levels of freestream turbulence, are identified and circumvented, delaying transition then is reduced to controlling the variety of possible linear modes: Tollmien-Schlichting modes, Mack modes, crossflow instabilities and Görtler instabilities. Tollmien-Schlichting instabilities dominate the transition process for two-dimensional boundary layers having $M a<4$, and are damped by increasing the Mach number, by wall cooling (in gases), and by the presence of favorable pressure-gradient. Contrast this to the Mack modes which dominate for two-dimensional hypersonic flows. Mack instabilities are also damped by increasing

the Mach number and by the presence of favorable pressure-gradient, but are destabilized by wall cooling. Crossflow and Görtler instabilities are caused by, respectively, the development of inflectional crossflow velocity profile and the presence of concave streamline curvature. Both of these instabilities are potentially harmful across the speed range, but are largely unaffected by Mach number and wall cooling. The crossflow modes are enhanced by favorable pressure-gradient, while the Görtler instabilities are insensitive. Suction suppresses, to different degrees, all the linear modes discussed in here.

\subsection{Convective and Absolute Instabilities}

In addition to grouping the different kinds of hydrodynamic instabilities as inviscid or viscous, one could also classify them as convective or absolute based on the linear response of the system to an initial localized impulse (Huerre and Monkewitz, 1990). A flow is convectively unstable if, at any fixed location, this response eventually decays in time. In other words, if all growing disturbances convect downstream from their source. Suppression of convective instabilities is particularly effective when applied near the point where the perturbations originate. If any of the growing disturbances has zero group velocity, the flow is absolutely unstable. This means that the local system response to an initial impulse grows in time. In this case, some of the growing disturbances can travel back upstream and continually disrupt the flow even after the 
initial disturbance is neutralized. Therefore, absolute instabilities are generally more dangerous and more difficult to control; nothing short of complete suppression will work. In some flows, for example two-dimensional blunt-body wakes, certain regions are absolutely unstable while others are convectively unstable.

\subsection{Classical Control Tools}

We end this section by listing several traditional flow control strategies that are either already in application or are market ready. Natural laminar flow (NLF) implies delaying transition via controlling the body shape to provide long runs of favorable pressure-gradient, and has been applied since the 1930s on airfoil sections to achieve lower skin-friction drag. Laminar flow control (LFC), in contrast, uses suction, wall heating/cooling and other active means of control to suppress the proper instability modes. Though well established in the laboratory and successfully field tested, routine application in the field of the variety of available LFC strategies is awaiting the removal of some technological hurdles related mostly to cost, maintenance and reliability issues (see, for example, Thibert et al., 1990; Wagner et al., 1990; Barnwell and Hussaini, 1992). Compliant coatings offer a rather simple method to delay laminar-to-turbulence transition as well as to interact favorably with a turbulent wall-bounded flow. In its simplest form, the technique is passive, relatively easy to apply to an existing vehicle or device, and perhaps not too expensive. The subject, though periodically discredited/redeemed, has been recently reviewed by Gad-el-Hak (1996), who argues that compliant coatings could be market ready with modest additional research effort.

Available techniques to reduce skin-friction drag in turbulent wall-bounded flows include LEBUs, riblets and polymer (for extensive references, see the three corresponding articles in the book edited by Bushnell and Hefner, 1990). The first two yield only modest drag reduction of the order of $10 \%$, while polymer additives result in substantial reduction of as much as $80 \%$. Polymers, appropriate only for hydrodynamic flows, are occasionally utilized in practical pipelines, for example in the 800-mile Trans-Alaskan Pipeline System (TAPS) that carries crude oil from Prudhoe Bay to the port of Valdez, but the main hurdle for using the strategy for external 
flows is the cost and weight of the additive to be carried onboard a surface ship or a submarine.

Of all the various types of shear-flow control now extant, control of flow separation, historically referred to as boundary-layer control (BLC), is probably the oldest and most economically important (Gad-el-Hak and Bushnell, 1991). Generally it is desired to postpone separation so that form drag is reduced, stall is delayed, lift is enhanced and pressure recovery is improved. However, in some instances it may be beneficial to provoke separation. For example, to improve the subsonic high-lift performance of an airfoil optimized for high-speed flight, a flap may be used to initiate leading-edge separation followed by reattachment.

Flow separation control is currently employed via vortex generators on the wings of most Boeing aircraft; via blown flaps on older generation supersonic fighters or leading-edge extensions and strakes on newer generations; and via passive bleed in the inlets of supersonic engines on, for example, the SR-71 and Concorde. Emerging control strategies for both steady and unsteady separation include transient suction and microblowing; these are described by, among others, Karim and Acharya (1994), Alrefai and Acharya (1995), and Roos (1996).

Future possibilities for aeronautical applications of flow separation control include providing structurally efficient alternatives to flaps or slats; cruise application on conventional takeoff and landing aircraft including BLC on thick spanloader wings; as well as cruise application on high-speed civil transports for favorable interference wave-drag reduction, increased leadingedge thrust, and enhanced fuselage and upper surface lift. In fact, much of the remaining gains to be made in aerodynamics appear to involve various types of flow control, including separated flow control. Typical, in some cases serious, problems associated with flow separation control include parasitic device drag or energy consumption; system weight, volume, complexity, reliability or cost; performance sensitivity to body attitude or orientation; and, especially in the case of the automobile, styling. 


\section{REACTIVE CONTROL}

\subsection{Targeted Control}

Numerous methods of flow control have already been successfully implemented in practical engineering devices. Yet, limitations exist for some familiar control techniques when applied to specific situations. For example, in attempting to reduce the drag or enhance the lift of a body having a turbulent boundary layer using global suction, the penalty associated with the control device often exceeds the saving derived from its use. What is needed is a way to reduce this penalty to achieve a more efficient control. Reactive control geared specifically towards manipulating the coherent structures in transitional and turbulent shear flows, though considerably more complicated than passive control or even predetermined active control, has the potential to do just that. As will be argued in this and the following three sections, future systems for control of transitional and turbulent flows in general and turbulent boundary layers in particular could greatly benefit from the merging of the science of chaos control and the technologies of microfabrication and neural networks. Such systems are envisaged as consisting of a large number of intelligent, communicative wall sensors and actuators arranged in a checkerboard pattern and targeted towards controlling certain quasi-periodic, dynamically significant coherent structures present in the wall region.

As discussed in Section 2, successful techniques to reduce the skin friction in a turbulent flow, such as polymers, particles or riblets, seem to act indirectly through local interaction with discrete turbulent structures, particularly small-scale eddies, within the flow. Common characteristics of all these methods are increased losses in the near-wall region, thickening of the buffer layer, and lowered production of Reynolds shear stress (Bandyopadhyay, 1986). Methods that act directly on the mean flow, such as suction or lowering of near-wall viscosity, also lead to inhibition of Reynolds stress. However, skin friction is increased when any of these velocityprofile modifiers is applied globally.

Could these seemingly inefficient techniques, e.g. global suction, be used more sparingly and be optimized to reduce their associated penalty? It appears that the more successful drag- 
reduction methods, e.g. polymers, act selectively on particular scales of motion and are thought to be associated with stabilization of the secondary instabilities. It is also clear that energy is wasted when suction or heating/cooling is used to suppress the turbulence throughout the boundary layer when the main interest is to affect a near-wall phenomenon. One ponders, what would become of wall turbulence if specific coherent structures are to be targeted, by the operator through a reactive control scheme, for modification? The myriad of organized structures present in all shear flows are instantaneously identifiable, quasi-periodic motions (Cantwell, 1981; Robinson, 1991). Bursting events in wall-bounded flows, for example, are both intermittent and random in space as well as time. The random aspects of these events reduce the effectiveness of a predetermined active control strategy. If such structures are nonintrusively detected and altered, on the other hand, net performance gain might be achieved. It seems clear, however, that temporal phasing as well as spatial selectivity would be required to achieve proper control targeted towards random events.

A rather simple version of the above idea is the selective suction technique which combines suction to achieve an asymptotic turbulent boundary layer and longitudinal riblets to fix the location of low-speed streaks. The feasibility of the selective suction as a drag-reducing concept has been demonstrated by Gad-el-Hak and Blackwelder (1989). Low-speed streaks were artificially generated in a laminar boundary layer using the method of Gad-el-Hak and Hussain (1986), and a hot-film probe was used to record the near-wall signature of the streaks. Using a feedforward control loop, an equivalent suction coefficient of $C_{q}=0.0006$ was sufficient to eliminate the artificial events and prevent bursting. This rate is five times smaller than the asymptotic suction coefficient for a corresponding turbulent boundary layer. If this result is sustained in a naturally developing turbulent boundary layer, a skin-friction reduction of close to $60 \%$ would be attained.

The recent numerical experiments of Choi et al. (1994) also validate the concept of targeting suction/injection to specific near-wall events in a turbulent channel flow. Based on complete interior flow information, their direct numerical simulations indicate a $20 \%$ net drag reduction accompanied by significant suppression of the near-wall structures and the Reynolds stress throughout the entire wall-bounded flow. When only wall information was used, a drag reduction 
of $6 \%$ was observed; a rather disappointing result considering that sensing and actuation took place at every grid point along the computational wall. In a practical implementation of this technique, even fewer wall sensors would perhaps be available, measuring only a small subset of the accessible information and thus requiring even more sophisticated control algorithms to achieve the same degree of success.

Time sequences of the numerical flow field of Choi et al. (1994) indicate the presence of two distinct drag-reducing mechanisms when selective suction/injection is used. First, deterring the sweep motion, without modifying the primary streamwise vortices above the wall, and consequently moving the high-shear regions from the surface to the interior of the channel, thus

directly reducing the skin friction. Secondly, changing the evolution of the wall vorticity layer by stabilizing and preventing lifting of the near-wall spanwise vorticity, thus suppressing a potential source of new streamwise vortices above the surface and interrupting a very important regeneration mechanism of turbulence.

\subsection{Reactive Feedback Control}

Moin and Bewley (1994) categorize reactive feedback control strategies by examining the extent to which they are based on the governing flow equations. Four categories are discerned: adaptive, physical model-based, dynamical systems-based, and optimal control (Figure 1). Note that except for adaptive control, the other three categories of reactive feedback control can also be used in the feedforward mode or the combined feedforward-feedback mode. Also, in a convective environment such as that for a boundary layer, a controller would perhaps combine feedforward and feedback information and may include elements from each of the four classifications. Each of the four categories is briefly described below.

Adaptive schemes attempt to develop models and controllers via some learning algorithm without regard to the details of the flow physics. System identification is performed independently of the flow dynamics or the Navier-Stokes equations which govern this dynamics. An adaptive controller tries to optimize a specified performance index by providing a control signal to an 
actuator. In order to update its parameters, the controller thus requires feedback information relating to the effects of its control. The most recent innovation in adaptive flow control schemes involves the use of neural networks which relate the sensor outputs to the actuator inputs through functions with variable coefficients and nonlinear, sigmoid saturation functions. The coefficients are updated using the so-called back-propagation algorithm, and complex control laws can be represented with a sufficient number of terms. Hand tuning is required, however, to achieve good convergence properties. The nonlinear adaptive technique has been successfully used by Fan $e t$ al. (1993) and Jacobson and Reynolds $(1993 ; 1995)$ to control, respectively, the transition process and the bursting events in turbulent boundary layers. We will return to this subject in Section 7.

Heuristic physical arguments can instead be used to establish effective control laws. That approach obviously will work only in situations in which the dominant physics are well understood. An example of this strategy is the active cancellation scheme, used by Gad-el-Hak and Blackwelder (1989) in a physical experiment and by Choi et al. (1994) in a numerical experiment, to reduce the drag by mitigating the effect of near-wall vortices. As mentioned earlier, the idea is to oppose the near-wall motion of the fluid, caused by the streamwise vortices, with an opposing wall control, thus lifting the high-shear region away from the surface and interrupting the turbulence regeneration mechanism.

Nonlinear dynamical systems theory allows turbulence to be decomposed into a small number of representative modes whose dynamics are examined to determine the best control law. The task is to stabilize the attractors of a low-dimensional approximation of a turbulent chaotic system. The best known example is the OGY method which, when applied to simpler, smallnumber of degrees of freedom systems, achieves stabilization with minute expenditure of energy. This and other chaos control strategies, especially as applied to the more complex turbulent flows, will be revisited in Section 5.2.

Finally, optimal control theory applied directly to the Navier-Stokes equations can be used to minimize a cost function in the space of the control. This strategy provides perhaps the most rigorous theoretical framework for flow control. In this method, feedback control laws are derived 
systematically for the most efficient distribution of control effort to achieve a desired goal. Abergel and Temam (1990) developed such optimal control theory for suppressing turbulence in a numerically simulated, two-dimensional Navier-Stokes flow, but their method requires an impractical full flow-field information. Choi et al. (1993) developed a more practical, wallinformation-only, sub-optimal control strategy which they applied to the one-dimensional stochastic Burgers equation. Later application of the sub-optimal control theory to a numerically simulated turbulent channel flow is reported by Moin and Bewley (1994).

\section{CHAOS CONTROL}

\subsection{Nonlinear Dynamical Systems Theory}

In the theory of dynamical systems, the so-called butterfly effect denotes sensitive dependence of nonlinear differential equations on initial conditions. The solution of such equations may be in the form of a strange attractor whose intrinsic structure contains a well-defined mechanism to produce a chaotic behavior without requiring random forcing. A question arises naturally: just as small disturbances can radically grow within a deterministic system to yield rich, unpredictable behavior, can minute adjustments to a system parameter be used to reverse the process and control, i.e. regularize, the behavior of a chaotic system? Recently, that question was answered in the affirmative theoretically as well as experimentally, at least for system orbits which reside on low-dimensional strange attractors (see the review by Lindner and Ditto, 1995). Before describing such strategies for controlling chaotic systems, we first summarize the recent attempts to construct a low-dimensional dynamical systems representation of turbulent boundary layers. Such construction is a necessary first step to be able to use chaos control methods for turbulent flows.

Boundary layer turbulence is described by a set of nonlinear partial differential equations and is characterized by an infinite number of degrees of freedom. This makes it rather difficult to model the turbulence using a dynamical systems approximation. The notion that a complex, infinite-dimensional flow can be decomposed into several low-dimensional subunits is, however, a natural consequence of the realization that quasi-periodic coherent structures dominate the 
dynamics of seemingly random turbulent shear flows. This implies that low-dimensional, localized dynamics can exist in formally infinite-dimensional extended systems--such as open turbulent flows. Reducing the flow physics to finite-dimensional dynamical systems enables a study of its behavior through an examination of the fixed points and the topology of their stable and unstable manifolds.

In one significant attempt the proper orthogonal, or Karhunen-Loève, decomposition method has been used to extract a low-dimensional dynamical system from experimental data of the wall region (Aubry et al. 1988; Aubry, 1990). Aubry et al. (1988) expanded the instantaneous velocity field of a turbulent boundary layer using experimentally determined eigenfunctions which are in the form of streamwise rolls. They expanded the Navier-Stokes equations using these optimally chosen, divergence-free, orthogonal functions, applied a Galerkin projection, and then truncated the infinite-dimensional representation to obtain a ten-dimensional set of ordinary differential equations. These equations represent the dynamical behavior of the rolls, and are shown to exhibit a chaotic regime as well as an intermittency due to a burst-like phenomenon. However, Aubry et al.'s ten-mode dynamical system displays a regular intermittency, in contrast both to that in actual turbulence as well as to the chaotic intermittency encountered by Pomeau and Manneville (1980) in which event durations are distributed stochastically. Nevertheless, the major conclusion of Aubry et al.'s study is that the bursts appear to be produced autonomously by the wall region even without turbulence, but are triggered by turbulent pressure signals from the outer layer. More recently, Berkooz et al. (1991) generalized the class of wall-layer models developed by Aubry et al. (1988) to permit uncoupled evolution of streamwise and cross-stream disturbances. Berkooz et al.'s results suggest that the intermittent events observed in Aubry et al. do not arise solely because of the effective closure assumption incorporated, but are rather rooted deeper in the dynamical phenomena of the wall region.

In addition to the reductionist viewpoint exemplified by the work of Aubry et al. (1988) and Berkooz et al. (1991), attempts have been made to determine directly the dimension of the attractors underlying specific turbulent flows. Again, the central issue here is whether or not 
turbulent solutions to the infinite-dimensional Navier-Stokes equations can be asymptotically described by a finite number of degrees of freedom. Grappin and Léorat (1991) computed the Lyapunov exponents and the attractor dimensions of two- and three-dimensional periodic turbulent flows without shear. They found that the number of degrees of freedom contained in the large scales establishes an upper bound for the dimension of the attractor. Deane and Sirovich (1991) and Sirovich and Deane (1991) numerically determined the number of dimensions needed to specify chaotic Rayleigh-Bénard convection over a moderate range of Rayleigh numbers, $R a$. They suggested that the intrinsic attractor dimension is $\mathrm{O}\left[R a^{2 / 3}\right]$.

The corresponding dimension in wall-bounded flows appears to be dauntingly high. Keefe et al. (1992) determined the dimension of the attractor underlying turbulent Poiseuille flows with spatially periodic boundary conditions. Using a coarse-grained numerical simulation, they computed a lower bound on the Lyapunov dimension of the attractor to be approximately 352 at a pressure-gradient Reynolds number of 3200. Keefe et al. (1992) argue that the attractor dimension in fully-resolved turbulence is unlikely to be much larger than 780. This suggests that periodic turbulent shear flows are deterministic chaos and that a strange attractor does underlie solutions to the Navier-Stokes equations. Temporal unpredictability in the turbulent Poiseuille flow is thus due to the exponential spreading property of such attractors. Although finite, the computed dimension invalidates the notion that the global turbulence can be attributed to the interaction of a few degrees of freedom. Moreover, in a physical channel or boundary layer, the flow is not periodic and is open. The attractor dimension in such case is not known but is believed to be even higher than the estimate provided by Keefe et al. for the periodic (quasi-closed) flow.

In contrast to closed, absolutely unstable flows, such as Taylor-Couette systems, where the number of degrees of freedom can be small, local measurements in open, convectively unstable flows, such as boundary layers, do not express the global dynamics, and the attractor dimension in that case may inevitably be too large to be determined experimentally. According to the estimate provided by Keefe et al. (1992), the colossal data required (about $10^{D}$, where $D$ is the attractor 
dimension) for measuring the dimension simply exceeds current computer capabilities.

\subsection{Chaos Control}

There is another question of greater relevance here. Given a dynamical system in the chaotic regime, is it possible to stabilize its behavior through some kind of active control? While other alternatives have been devised (e.g., Fowler, 1989; Hübler and Lüscher, 1989; Huberman, 1990; Huberman and Lumer, 1990), the recent method proposed by workers at the University of Maryland (Ott et al., 1990a; 1990b; Shinbrot et al., 1990; 1992a; 1992b; 1992c; Romeiras et al., 1992) promises to be a significant breakthrough. Comprehensive reviews and bibliographies of the emerging field of chaos control can be found in the articles by Shinbrot et al. (1993), Shinbrot (1993; 1995), and Lindner and Ditto (1995).

Ott et al. (1990a) demonstrated, through numerical experiments with the Henon map, that it is possible to stabilize a chaotic motion about any pre-chosen, unstable orbit through the use of relatively small perturbations. The procedure consists of applying minute time-dependent perturbations to one of the system parameters to control the chaotic system around one of its many unstable periodic orbits. In this context, targeting refers to the process whereby an arbitrary initial condition on a chaotic attractor is steered toward a prescribed point (target) on this attractor. The goal is to reach the target as quickly as possible using a sequence of small perturbations (Kostelich et al., 1993a).

The success of the Ott-Grebogi-Yorke's (OGY) strategy for controlling chaos hinges on the fact that beneath the apparent unpredictability of a chaotic system lies an intricate but highly ordered structure. Left to its own recourse, such a system continually shifts from one periodic pattern to another, creating the appearance of randomness. An appropriately controlled system, on the other hand, is locked into one particular type of repeating motion. With such a reactive control the dynamical system becomes one with a stable behavior. The OGY-method can be simply illustrated by the schematic in Figure 3. The state of the system is represented as the intersection of a stable manifold and an unstable one. The control is applied intermittently whenever the system departs from the stable manifold by a prescribed tolerance, otherwise the control is shut off. The control 
attempts to put the system back onto the stable manifold so that the state converges toward the desired trajectory. Unmodeled dynamics cause noise in the system and a tendency for the state to wander off in the unstable direction. The intermittent control prevents that and the desired trajectory is achieved. This efficient control is not unlike trying to balance a ball in the center of a horse saddle (Moin and Bewley, 1994). There is one stable direction (front/back) and one unstable direction (left/right). The restless horse is the umodeled dynamics, intermittently causing the ball to move in the wrong direction. The OGY-control needs only be applied, in the most direct manner possible, whenever the ball wanders off in the left/right direction.

The OGY-method has been successfully applied in a relatively simple experiment by Ditto et al. (1990) and Ditto and Pecora (1993) at the Naval Surface Warfare Center, in which reverse chaos was obtained in a parametrically driven, gravitationally buckled, amorphous magnetoelastic ribbon. Garfinkel et al. (1992) applied the same control strategy to stabilize drug-induced cardiac arrhythmias in sections of a rabbit ventricle. Other extensions, improvements and applications of the OGY-strategy include higher-dimensional targeting (Auerbach et al., 1992; Kostelich et al., 1993b), controlling chaotic scattering in Hamiltonian (i.e., nondissipative, area conservative) systems (Lai et al., 1993a; 1993b), synchronization of identical chaotic systems that govern communication, neural or biological processes (Lai and Grebogi, 1993), use of chaos to transmit information (Hayes et al., 1993), control of transient chaos (Lai et al., 1994), and taming spatiotemporal chaos using a sparse array of controllers (Chen et al., 1993; Qin et al., 1994; Auerbach, 1994).

In a more complex system, such as a turbulent boundary layer, there exist numerous interdependent modes and many stable as well as unstable manifolds (directions). Factors that make turbulence control a challenging task are the potentially quite large perturbations caused by the unmodeled dynamics of the flow, the non-stationary nature of the desired dynamics, and the complexity of the saddle shape describing the dynamics of the different modes. Nevertheless, the OGY-control strategy has several advantages that are of special interest in the control of turbulence: the mathematical model for the dynamical system need not be known; only small changes in the 
control parameter are required; and noise can be tolerated (with appropriate penalty).

Recently, Keefe (1993a; 1993b) made a useful comparison between two nonlinear control strategies as applied to fluid problems. Ott-Grebogi-Yorke's feedback method described above and the model-based control strategy originated by Hübler (see, for example, Hübler and Lüscher, 1989; Lüscher and Hübler, 1989), the H-method. Both novel control methods are essentially a generalization of the classical perturbation cancellation technique: apply a prescribed forcing to subtract the undesired dynamics and impose the desired one. The OGY-strategy exploits the sensitivity of chaotic systems to stabilize existing periodic orbits and steady states. Some feedback is needed to steer the trajectories toward the chosen fixed point, but the required control signal is minuscule. In contrast, Hübler's scheme does not explicitly make use of the system sensitivity. It produces general control response (periodic or aperiodic) and needs little or no feedback, but its control inputs are generally large. The OGY-strategy exploits the nonlinearity of a dynamical system; indeed the presence of a strange attractor and the extreme sensitivity of the dynamical system to initial conditions are essential to the success of the method. In contrast, the H-method works equally for both linear and nonlinear systems.

Keefe (1993a) first examined numerically the two schemes as applied to fully-developed and transitional solutions of the Ginzburg-Landau equation, an evolution equation that governs the initially weakly nonlinear stages of transition in several flows and that possesses both transitional and fully-chaotic solutions. The Ginzburg-Landau equation has solutions that display either absolute or convective instabilities, and is thus a reasonable model for both closed and open flows. Keefe's main conclusion is that control of nonlinear systems is best obtained by making maximum use possible of the underlying natural dynamics. If the goal dynamics is an unstable nonlinear solution of the equation and the flow is nearby at the instant control is applied, both methods perform reliably and at low-energy cost in reaching and maintaining this goal. Predictably, the performance of both control strategies degrades due to noise and the spatially discrete nature of realistic forcing. Subsequently, Keefe (1993b) extended the numerical experiment in an attempt to reduce the drag in a channel flow with spatially periodic boundary conditions. The OGY-method 
reduces the skin friction to $60-80 \%$ of the uncontrolled value at a mass-flux Reynolds number of 4408. The H-method fails to achieve any drag reduction when starting from a fully-turbulent initial condition but shows potential for suppressing or retarding laminar-to-turbulent transition. Keefe (1993a) suggests that the H-strategy might be more appropriate for boundary layer control, while the OGY-method might best be used for channel flows.

It is also relevant to note here the work of Bau and his colleagues at the University of Pennsylvania (Singer et al., 1991; Wang et al., 1992), who devised a feedback control to stabilize (relaminarize) the naturally occurring chaotic oscillations of a toroidal thermal convection loop heated from below and cooled from above. Based on a simple mathematical model for the thermosyphon, Bau and his colleagues constructed a reactive control system that was used to alter significantly the flow characteristics inside the convection loop. Their linear control strategy, perhaps a special version of the OGY's chaos control method, consists simply of sensing the deviation of fluid temperatures from desired values at a number of locations inside the thermosyphon loop and then altering the wall heating either to suppress or to enhance such deviations. Wang et al. (1992) also suggested extending their theoretical and experimental method to more complex situations such as those involving Bénard convection (Tang and Bau, 1993a; 1993b). Hu and Bau (1994) used a similar feedback control strategy to demonstrate that the critical Reynolds number for the loss of stability of planar Poiseuille flow can be significantly increased or decreased.

Other attempts to use low-dimensional dynamical systems representation for flow control include the work of Berkooz et al. (1993), Corke et al. (1994), and Coller et al. (1994a; 1994b). Berkooz et al. (1993) applied techniques of modern control theory to estimate the phase-space location of dynamical models of the wall-layer coherent structures, and used these estimates to control the model dynamics. Since discrete wall-sensors provide incomplete knowledge of phasespace location, Berkooz et al. maintain that a nonlinear observer, which incorporates past information and the equations of motion into the estimation procedure, is required. Using an extended Kalman filter, they achieved effective control of a bursting pair of rolls with the 
equivalent of two wall-mounted shear sensors. Corke et al. (1994) used a low-dimensional dynamical system based on the proper orthogonal decomposition to guide control experiments for an axisymmetric jet. By sensing the downstream velocity and actuating an array of miniature speakers located at the lip of the jet, their feedback control succeeded in converting the near-field instabilities from spatial-convective to temporal-global. Coller et al. (1994a; 1994b) developed a feedback control strategy for strongly nonlinear dynamical systems, such as turbulent flows, subject to small random perturbations that kick the system intermittently from one saddle point to another along heteroclinic cycles. In essence, their approach is to use local, weakly nonlinear feedback control to keep a solution near a saddle point as long as possible, but then to let the natural, global nonlinear dynamics runs its course when bursting (in a low-dimensional model) does occur. Though conceptually related to the OGY-strategy, Coller et al.'s method does not actually stabilize the state but merely holds the system near the desired point longer than it would otherwise stay.

Shinbrot and Ottino (1993a; 1993b) offer yet another strategy presumably most suited for controlling coherent structures in area-preserving turbulent flows. Their geometric method exploits the premise that the dynamical mechanisms which produce the organized structures can be remarkably simple. By repeated stretching and folding of "horseshoes" which are present in chaotic systems, Shinbrot and Ottino have demonstrated numerically as well as experimentally the ability to create, destroy and manipulate coherent structures in chaotic fluid systems. The key idea to create such structures is to intentionally place folds of horseshoes near low-order periodic points. In a dissipative dynamical system, volumes contract in state space and the co-location of a fold with a periodic point leads to an isolated region that contracts asymptotically to a point. Provided that the folding is done properly, it counteracts stretching. Shinbrot and Ottino (1993a) applied the technique to three prototypical problems: a one-dimensional chaotic map; a twodimensional one; and a chaotically advected fluid. 


\section{MICROFABRICATION}

Manufacturing processes that can create microscopic machinery are termed microfabrications. In this emerging technology, under intensive development only since 1990, electronic and mechanical components are combined on a single silicon chip. Sensors for pressure, temperature, velocity, mass flow or sound are currently combined with motors, electrostatic actuators, pneumatic actuators, pumps, valves, gears or tweezers on single programmable elements of typical size $\mathrm{O}[10 \mu]$. Microelectromehanical systems (or MEMS) have been constructed and tested during the last few years at numerous laboratories. The new Journal of Microelectromehanical Systems is dedicated to this technology, and the older Sensors and Actuators is increasingly allotting more of its pages to MEMS. Entire sessions in scientific meetings have been increasingly assigned to MEMS applications in fluid mechanics (see, for example, the presentations by McMichael, Tai, Mehregany, Mastrangelo, and Yun, all made at the AIAA Third Shear Flow Control Conference, Orlando, Florida, 6-9 July 1993, and the volumes edited by Bandyopadhyay et el., 1994, and Breuer et al., 1996). Recent reviews of the use, or potential use, of MEMS in flow control include those by McMichael (1996), and Mehregany et al. (1996).

MEMS would be ideal for the reactive flow control concept advocated in the present paper. Methods of flow control targeted towards specific coherent structures involve nonintrusive detection and subsequent modulation of events that occur randomly in space and time. To achieve proper targeted control of these quasi-periodic vortical events, temporal phasing as well as spatial selectivity are required. Practical implementation of such an idea necessitates the use of a large number of intelligent, communicative wall sensors and actuators arranged in a checkerboard pattern. Gad-el-Hak (1994) provides estimates for the number and characteristics of such elements required to modulate the turbulent boundary layer which develops along a typical commercial aircraft or nuclear submarine. An upper-bound number to achieve total turbulence suppression is about one million sensors/actuators per square meter of the surface.

The sensors would be expected to measure the amplitude, location, and phase or frequency 
of the signals impressed upon the wall by incipient bursting events. Instantaneous wall-pressure or wall-shear stress can be sensed, for example. The normal or in-plane motion of a minute membrane is proportional to the respective point force of primary interest. For measuring wall pressure, microphone-like devices respond to the motion of a vibrating surface membrane or an internal elastomer. Several types are available including variable-capacitance (condenser or electret), ultrasonic, optical (e.g., optical-fiber and diode-laser), and piezoelectric devices (see, for example, Löfdahl et al., 1993; 1994). A potentially useful technique for our purposes has been recently tried at MIT (J. H. Haritonidis, private communications). An array of extremely small (0.2 $\mathrm{mm}$ in diameter) laser-powered microphones (termed picophones) was machined in silicon using integrated circuit fabrication techniques, and was used for field measurement of the instantaneous surface pressure in a turbulent boundary layer.

Actuators are expected to produce a desired change in the targeted coherent structures. The local acceleration action needed to stabilize an incipient bursting event can be in the form of adaptive wall, transpiration or wall heat transfer. Traveling surface waves can be used to modify a locally convecting pressure gradient such that the wall motion follows that of the coherent event causing the pressure change. Surface motion in the form of a Gaussian hill with height $y^{+}=\mathrm{O}[10]$ should be sufficient to suppress typical incipient bursts (Lumley, 1991). Such timedependent alteration in wall geometry can be generated by driving a flexible skin using an array of piezoelectric devices (dilate or contract depending on the polarity of current passing through them), electromagnetic actuators, magnetoelastic ribbons (made of nonlinear materials that change their stiffness in the presence of varying magnetic fields), or Terfenol-d rods (a novel metal composite, developed at Grumman Corporation, which changes its length when subjected to a magnetic field). Note should also be made of other exotic materials that can be used for actuation. For example, electrorheological fluids (Halsey and Martin, 1993) instantly solidify when exposed to an electric field, and may thus be useful for the present application. Recently constructed microactuators specifically designed for flow control include those by Wiltse and Glezer (1993), James et al. (1994), Jacobson and Reynolds (1995), and Vargo and Muntz (1996). 
Suction/injection at many discrete points can be achieved by simply connecting a large number of minute streamwise slots, arranged in a checkerboard pattern, to a low-pressure/high-pressure reservoir located underneath the working surface. The transpiration through each individual slot is turned on and off using a corresponding number of independently controlled microvalves. Alternatively, positive-displacement or rotary micropumps (see, for example, Sen et al., 1996; Sharatchandra et al., 1996) can be used for blowing or sucking fluid through small holes/slits. Based on the results of Gad-el-Hak and Blackwelder (1989), equivalent suction coefficients of about 0.0006 should be sufficient to stabilize the near-wall region. Assuming that the skin-friction coefficient in the uncontrolled boundary layer is $C_{f}=0.003$, and assuming further that the suction used is sufficient to establish an asymptotic boundary layer ( $d \theta / d x=0$, where $\theta$ is the momentum thickness), the skin friction in the reactively controlled case is then $C_{f}=0+2 C_{q}=0.0012$, or $40 \%$ of the original value. The net benefit will, of course, be reduced by the energy expenditure of the suction pump (or micropumps) as well as the array of microsensors and microvalves.

Finally, if the bursting events are to be eliminated by lowering the near-wall viscosity, direct electric-resistance heating can be used in liquid flows and thermoelectric devices based on the Peltier effect can be used for cooling in the case of gaseous boundary layers. The absolute viscosity of water at $20^{\circ} \mathrm{C}$ decreases by approximately $2 \%$ for each $1^{\circ} \mathrm{C}$ rise in temperature, while for room-temperature air, $\mu$ decreases by approximately $0.2 \%$ for each $1^{\circ} \mathrm{C}$ drop in temperature. The streamwise momentum equation written at the wall can be used to show that a suction coefficient of 0.0006 has approximately the same effect on the wall-curvature of the instantaneous velocity profile as a surface heating of $2^{\circ} \mathrm{C}$ in water or a surface cooling of $40^{\circ} \mathrm{C}$ in air (Liepmann and Nosenchuck, 1982; Liepmann et al., 1982).

Sensors and actuators of the types discussed above can be combined on individual electronic chips using microfabrication technology. The chips can be interconnected in a communication network that is controlled by a massively parallel computer or a self-learning neural 
network (Section 7). Factors to be considered in an eventual field application of chips produced using microfabrication processes include sensitivity of sensors, sufficiency and frequency response of actuators' action, fabrication of large arrays at affordable prices, survivability in the hostile field environment, and energy required to power the sensors/actuators. As argued by Gadel-Hak (1994), sensor/actuator chips currently produced are small enough for typical field application, and they can be programmed to provide a sufficiently large/fast action in response to a certain sensor output (see also Jacobson and Reynolds, 1995). Present prototypes are, however, still quite expensive as well as delicate. But so was the transistor when first introduced! It is hoped that the unit price of future sensor/actuator elements would follow the same dramatic trends witnessed in case of the simple transistor and even the much more complex integrated circuit.

\section{NEURAL NETWORKS}

Biologically inspired neural networks are finding increased applications in many fields of science and technology. Modeling of complex dynamical systems, adaptive noise canceling in telephones and modems, bomb sniffers, mortgage-risk evaluators, sonar classifiers, and word recognizers are but a few of existing usages of neural nets. The book by Nelson and Illingworth (1991) provides a lucid introduction to the field, and the review article by Antsaklis (1993) focuses

on the use of neural nets for the control of complex dynamical systems. For flow control applications, neural networks offer the possibility of adaptive controllers that are simpler and potentially less sensitive to parameter variations as compared to conventional controllers. Moreover, if a colossal number of sensors and actuators is to be used, the massively parallel computational power of neural nets will surely be needed for real-time control.

The basic elements of a neural network are schematically shown in Figure 4. Several inputs are connected to the nodes (neurons or processing elements) that form the input layer. There are one or more hidden layers, followed by an output layer. Note that the number of connections is higher than the total number of nodes. Both numbers are chosen based on the particular application and can be arbitrarily large for complex tasks. Simply put, the multi-task job 
of each processing element is to evaluate each of the input signals to that particular element, calculate the weighted sum of the combined inputs, compare that total to some threshold level, and finally determine what the output should be. The various weights are the adaptive coefficients which vary dynamically as the network learns to perform its assigned task; some inputs are more important than others. The threshold, or transfer, function is generally nonlinear; the most common one being the continuous sigmoid, or S-shaped, curve, which approaches a minimum and maximum value at the asymptotes. If the sum of the weighted inputs is larger than the threshold value, the neuron generates a signal; otherwise no signal is fired. Neural networks can operate in feedforward or feedback mode. $\S$ Complex systems, for which dynamical equations may not be known or may be too difficult to solve, can be modeled using neural nets.

For flow control, neural networks provide convenient, fast, nonlinear adaptive algorithms to relate sensor outputs to actuator inputs via variable-coefficient functions and nonlinear, sigmoid saturation functions. With no prior knowledge of the pertinent dynamics, a self-learning neural network develops a model for that dynamics through observations of the applied control and sensed measurements. The network is by nature nonlinear and can therefore better handle nonlinear dynamical systems, a difficult task when classical, linear or weakly nonlinear, control strategies are attempted. The feedforward type of neural network acts as a nonlinear filter forming an output from a set of input data. The output can then be compared to some desired output, and the difference (error) is typically used in a back-propagation algorithm which updates the network parameters.

The number of researchers using neural networks to control fluid flows is growing rapidly. In here, we provide only a small sample. Using a pre-trained neural network, Fan et al. (1993) conducted a conceptual reactive flow control experiment to delay laminar-to-turbulence transition. Numerical simulations of their flow control system demonstrate almost complete cancellation of single and multiple artificial wave disturbances. Their controller also successfully attenuated a

\footnotetext{
$\S$ Note that this terminology refers to the direction of information through the network. When a neural net is used as a controller, the overall control loop is, however, a feedback, closed loop: the self-learning network dynamically updates its various parameters by comparing its output to a desired output, thus requiring feedback information relating to the effect of its control.
} 
natural disturbance signal with developing wave packets from an actual wind-tunnel experiment. Jacobson and Reynolds $(1993 ; 1995)$ used neural networks to minimize the boundary velocity gradient of three model flows: the one-dimensional stochastic Burgers equation; a twodimensional computational model of the near-wall region of a turbulent boundary layer; and a realtime turbulent flow with a spanwise array of wall actuators together with upstream and downstream wall-sensors. For all three problems, the neural network successfully learned about the flow and developed into proficient controllers. However, for the laboratory experiments, Jacobson and Reynolds (1995) report that the neural network training time was much longer and the performance was no better than a simpler ad hoc controller that they developed. Jacobson and Reynolds emphasize that alternative neural net configurations and convergence algorithms may, however, greatly improve the network performance.

Using the angle of attack and angular velocity as inputs, Faller et al. (1994) trained a neural network to model the measured unsteady surface pressure over a pitching airfoil (see also Schreck et al., 1995). Following training and using the instantaneous angle of attack and pitch rate as the only inputs, their network was able to accurately predict the surface pressure topology as well as the time-dependent aerodynamic forces and moments. The model was then used to develop a neural network controller for wing-motion actuator signals which in turn provided direct control of the lift-to-drag ratio across a wide range of time-dependent motion histories. Most recently, Kawthar-Ali and Acharya (1996) developed a neural network controller for use in suppressing the dynamic-stall vortex that periodically develops in the leading edge of a pitching airfoil. Based on the current state of the unsteady pressure field, their control system specified the optimum amount of leading-edge suction to achieve complete vortex suppression.

\section{CLOSING REMARKS}

The present article reviewed the important advances in the field of flow control that took place during the past few years. An attempt has been made to place the field in a unifying framework and to properly categorize the different control strategies. Developments in chaos 
control, microfabrication and neural networks are making it more feasible to perform reactive control of turbulent flows. Field applications, however, have to await further progress in those three areas. Other less complex control schemes, passive as well as active, are more market ready and are also witnessing resurgence of interest.

As is clear from this brief review, there is no lack of flow control methods to achieve a particular goal for free as well as wall-bounded flows across the entire range of Mach and Reynolds numbers. Ranging from simple to complex, from inexpensive to expensive, from passive to active to reactive, and from market ready to futuristic, the fluid engineer has a great variety of control devices to choose from. Flow control is most effective when applied near the transition or separation points; in other words, near the critical flow regimes where flow instabilities magnify quickly. Therefore, delaying/advancing laminar-to-turbulence transition and preventing/provoking separation can be readily accomplished. To reduce the skin-friction drag in a non-separating turbulent boundary layer, where the mean flow is quite stable, is a more challenging problem.

Market-ready techniques include passive and predetermined active control. Shaping, suction, heating/cooling and compliant coatings can be used to delay transition by an order of magnitude in Reynolds number, and, except for the latter technique, can also be used to prevent boundary layer separation. The use of polymers, microbubbles, riblets and LEBUs can lead to skin-friction reduction in turbulent boundary layers. Numerous other techniques are available to reduce form drag, induced drag and wave drag. Remaining issues for field application of marketready techniques include cost, maintenance and reliability. Potential further improvements in classical flow control techniques will perhaps involve combining more than one technique aiming at achieving a favorable effect that is greater than the sum. Examples include combining suction or polymer injection with riblets for increased effectiveness and saving. Due to its obvious difficulties, synergism has not been extensively studied in the past but deserves future consideration.

Substantial gains are potentially possible when reactive flow control strategies are used. 
Particularly for turbulent flows, reactive control requires large number of sensors and actuators and will not be possible until the technology for manufacturing inexpensive, robust microsensors and microactuators becomes available. Autonomous control algorithms and associated computers to handle the required colossal data in real time must also be developed. Further research is needed in dynamical systems theory particularly chaos control, microelectromechanical systems, and alternative neural network configurations, convergence algorithms and distributed flow control. The difficulties are daunting but the potential payoffs are enormous.

In parting, it might be worth recalling that a mere $10 \%$ reduction in the total drag of an aircraft translates into a saving of $\$ 1$ billion in annual fuel cost for the commercial fleet in the United States alone. Contrast this benefit to the annual worldwide cost of perhaps a few million dollars for all basic research in the broad field of flow control. Taming turbulence, though difficult, will pay for itself in gold.

A parting verse from William Shakespeare's The Taming of the Shrew:

Hortensio (a gentleman of Padua): Now go thy ways, thou hast tam'd a curst shrew.

Lucentio (a gentleman of Pisa): 'Tis a wonder, by your leave, she will be tam'd so.

Different versions of the present paper have been delivered at the ASME Symposium on the Control of Shear and Convective Flows, Atlanta, Georgia, 17-22 November 1996, and at the International Congress of Fluid Dynamics and Propulsion, Cairo, Egypt, 29-31 December 1996. 


\section{REFERENCES}

Abergel, F., and Temam, R. (1990) "On Some Control Problems in Fluid Mechanics," Theor. Comput. Fluid Dyn. 1, pp. 303-325.

Alrefai, M., and Acharya, M. (1995) "Controlled Leading-Edge Suction for the Management of Unsteady Separation over Pitching Airfoils," AIAA Paper No. 95-2188, Washington, D.C.

Antsaklis, P.J. (1993) "Control Theory Approach," in Mathematical Approaches to Neural Networks, ed. J.G. Taylor, pp. 1-23, Elsevier, Amsterdam.

Aubry, N. (1990) "Use of Experimental Data for an Efficient Description of Turbulent Flows," Appl. Mech. Rev. 43, pp. S240-S245.

Aubry, N., Holmes, P., Lumley, J.L., and Stone, E. (1988) "The Dynamics of Coherent Structures in the Wall Region of a Turbulent Boundary Layer," J. Fluid Mech. 192, pp. 115-173.

Auerbach, D. (1994) "Controlling Extended Systems of Chaotic Elements," Phys. Rev. Lett. 72, pp. 1184-1187.

Auerbach, D., Grebogi, C., Ott, E., and Yorke, J.A. (1992) "Controlling Chaos in High Dimensional Systems," Phys. Rev. Lett. 69, pp. 3479-3482.

Bandyopadhyay, P.R. (1986) "Review--Mean Flow in Turbulent Boundary Layers Disturbed to Alter Skin Friction," J. Fluids Eng. 108, pp. 127-140. 
Bandyopadhyay, P.R., Breuer, K.S., and Blechinger, C.J. (editors) (1994) Application of Microfabrication to Fluid Mechanics, FED Vol. 197, American Society of Mechanical Engineering, New York.

Barnwell, R.W., and Hussaini, M.Y. (editors) (1992) Natural Laminar Flow and Laminar Flow Control, Springer-Verlag, New York.

Berkooz, G., Fisher, M., and Psiaki, M. (1993) "Estimation and Control of Models of the Turbulent Wall Layer," Bul. Am. Phys. Soc. 38, p. 2197.

Berkooz, G., Holmes, P., and Lumley, J.L. (1991) "Intermittent Dynamics in Simple Models of the Turbulent Wall Layer," J. Fluid Mech. 230, pp. 75-95.

Breuer, K.S., Gad-el-Hak, M., and Bandyopadhyay, P.R. (editors) (1996) Application of Silicon Microfabrication to Fluid Mechanics, FED Vol. ..., American Society of Mechanical Engineering, New York.

Bushnell, D.M. (1994) "Viscous Drag Reduction in Aeronautics," Proceedings of the Nineteenth Congress of the International Council of the Aeronautical Sciences, vol. 1, pp. XXXIII-LVI, Paper No. ICAS-94-0.1, American Institute of Aeronautics and Astronautics, Washington, D.C.

Bushnell, D.M., and Hefner, J.N. (editors) (1990) Viscous Drag Reduction in Boundary Layers, Progress in Astronautics and Aeronautics, vol. 123, American Institute of Aeronautics and Astronautics, Washington, D.C.

Bushnell, D.M., and McGinley, C.B. (1989) "Turbulence Control in Wall Flows," Annu. Rev. Fluid Mech. 21, pp. 1-20. 
Cantwell, B.J. (1981) "Organized Motion in Turbulent Flow," Annu. Rev. Fluid Mech. 13, pp. $457-515$.

Chen, C.-C., Wolf, E.E., and Chang, H.-C. (1993) "Low-Dimensional Spatiotemporal Thermal Dynamics on Nonuniform Catalytic Surfaces," J. Phys. Chemistry 97, pp. 1055-1064.

Choi, H., Moin, P., and Kim, J. (1994) "Active Turbulence Control for Drag Reduction in WallBounded Flows," J. Fluid Mech. 262, pp. 75-110.

Choi, H., Temam, R., Moin, P., and Kim, J. (1993) "Feedback Control for Unsteady Flow and its Application to the Stochastic Burgers Equation," J. Fluid Mech. 253, pp. 509-543.

Coller, B.D., Holmes, P., and Lumley, J.L. (1994a) "Control of Bursting in Boundary Layer Models," Appl. Mech. Rev. 47, pp. S139-S143.

Coller, B.D., Holmes, P., and Lumley, J.L. (1994b) "Control of Noisy Heteroclinic Cycles," Physica D 72, pp. 135-160.

Corke, T.C., Glauser, M.N., and Berkooz, G. (1994) "Utilizing Low-Dimensional Dynamical Systems Models to Guide Control Experiments," Appl. Mech. Rev. 47, pp. S132-S138.

Deane, A.E., and Sirovich, L. (1991) "A Computational Study of Rayleigh-Bénard Convection. Part 1. Rayleigh-Number Scaling," J. Fluid Mech. 222, pp. 231-250.

Ditto, W.L., and Pecora, L.M. (1993) "Mastering Chaos," Scientific American 269, August, pp. 78-84. 
Ditto, W.L., Rauseo, S.N., and Spano, M.L. (1990) "Experimental Control of Chaos," Phys. Rev. Lett. 65, pp. 3211-3214.

Faller, W.E., Schreck, S.J., and Luttges, M.W. (1994) "Real-Time Prediction and Control of Three-Dimensional Unsteady Separated Flow Fields Using Neural Networks," AIAA Paper No. 94-0532, Washington, D.C.

Fan, X., Hofmann, L., and Herbert, T. (1993) "Active Flow Control with Neural Networks," AIAA Paper No. 93-3273, Washington, D.C.

Fiedler, H.E., and Fernholz, H.-H. (1990) "On Management and Control of Turbulent Shear Flows," Prog. Aerospace Sci. 27, pp. 305-387.

Fiedler, H.E., Glezer, A., and Wygnanski, I. (1988) "Control of Plane Mixing Layer: Some Novel Experiments," in Current Trends in Turbulence Research, eds. H. Branover, M. Mond and Y. Unger, Progress in Astronautics and Aeronautics, vol. 112, pp. 30-64, AIAA, Washington, D.C.

Fowler, T.B. (1989) "Application of Stochastic Control Techniques to Chaotic Nonlinear Systems," IEEE Trans. Autom. Control 34, pp. 201-205.

Gad-el-Hak, M. (1989) "Flow Control," Appl. Mech. Rev. 42, pp. 261-293.

Gad-el-Hak, M. (1990) "Control of Low-Speed Airfoil Aerodynamics," AIAA J. 28, pp. $1537-1552$. 
Gad-el-Hak, M. (1994) "Interactive Control of Turbulent Boundary Layers: A Futuristic Overview," AIAA J. 32, pp. 1753-1765.

Gad-el-Hak, M. (1996) "Compliant Coatings: a Progress Report," Appl. Mech. Rev. 49, no. 10, part 2, pp. S147-S157.

Gad-el-Hak, M., and Blackwelder, R.F. (1989) "Selective Suction for Controlling Bursting Events in a Boundary Layer," AIAA J. 27, pp. 308-314.

Gad-el-Hak, M., and Bushnell, D.M. (1991) "Separation Control: Review," J. Fluids Eng. 113, pp. 5-30.

Gad-el-Hak, M., and Hussain, A. K. M. F. (1986) "Coherent Structures in a Turbulent Boundary Layer. Part 1. Generation of 'Artificial' Bursts," Phys. Fluids 29, pp. 2124-2139.

Garfinkel, A., Spano, M.L., Ditto, W.L., and Weiss, J.N. (1992) "Controlling Cardiac Chaos," Science 257, pp. 1230-1235.

Grappin, R., and Léorat, J. (1991) "Lyapunov Exponents and the Dimension of Periodic Incompressible Navier-Stokes Flows: Numerical Measurements," J. Fluid Mech. 222, pp. 61-94.

Gutmark, E., and Ho, C.-M. (1986) "Visualization of a Forced Elliptical Jet," AIAA J. 24, pp. 684-685.

Gutmark, E.J., Schadow, K.C., and Yu, K.H. (1995) "Mixing Enhancement in Supersonic Free Shear Flows," Annu. Rev. Fluid Mech. 27, pp. 375-417. 
Halsey, T.C., and Martin, J.E. (1993) "Electrorheological Fluids," Scientific American 269, October, pp. 58-64.

Hough, G.R. (editor) (1980) Viscous Flow Drag Reduction, Progress in Astronautics and Aeronautics, vol. 72, American Institute of Aeronautics and Astronautics, New York.

Hu, H.H. and Bau, H.H. (1994) "Feedback Control to Delay or Advance Linear Loss of Stability in Planar Poiseuille Flow," Proc. Roy. Soc. Lond. A 447, pp. 299-312.

Huberman, B. (1990) "The Control of Chaos," Proc. Workshop on Applications of Chaos, 4-7 December, San Francisco, California.

Huberman, B.A., and Lumer, E. (1990) "Dynamics of Adaptive Systems," IEEE Trans. Circuits Syst. 37, pp. 547-550.

Hübler, A., and Lüscher, E. (1989) "Resonant Stimulation and Control of Nonlinear Oscillators," Naturwissenschaften 76, pp. 67-69.

Huerre, P., and Monkewitz, P.A. (1990) "Local and Global Instabilities in Spatially Developing Flows," Annu. Rev. Fluid Mech. 22, pp. 473-537.

Jacobson, S.A., and Reynolds, W.C. (1993) "Active Control of Boundary Layer Wall Shear Stress Using Self-Learning Neural Networks," AIAA Paper No. 93-3272, Washington, D.C.

Jacobson, S.A., and Reynolds, W.C. (1995) "An Experimental Investigation towards the Active Control of Turbulent Boundary Layers," Department of Mechanical Engineering Report No. 
TF-64, Stanford University, Stanford, California. 
James, R.D., Jacobs, J.W., and Glezer, A. (1994) "Experimental Investigation of a Turbulent Jet Produced by an Oscillating Surface Actuator," Appl. Mech. Rev. 47, pp. S127-S1131.

Karim, M.A., and Acharya, M. (1994) "Control of the Dynamic-Stall Vortex over a Pitching Airfoil by Leading-Edge Suction," AIAA J. 32, pp. 1647-1655.

Kawthar-Ali, M.H., and Acharya, M. (1996) "Artificial Neural Networks for Suppression of the Dynamic-Stall Vortex over Pitching Airfoils," AIAA Paper No. 96-0540, Washington, D.C.

Keefe, L.R. (1993a) "Two Nonlinear Control Schemes Contrasted in a Hydrodynamic Model," Phys. Fluids A 5, pp. 931-947.

Keefe, L.R. (1993b) "Drag Reduction in Channel Flow Using Nonlinear Control," AIAA Paper No. 93-3279, Washington, D.C.

Keefe, L.R., Moin, P., and Kim, J. (1992) "The Dimension of Attractors Underlying Periodic Turbulent Poiseuille Flow," J. Fluid Mech. 242, pp. 1-29.

Kostelich, E.J., Grebogi, C., Ott, E., and Yorke, J.A. (1993a) "Targeting from Time Series," Bul. Am. Phys. Soc. 38, p. 2194.

Kostelich, E.J., Grebogi, C., Ott, E., and Yorke, J.A. (1993b) "Higher-Dimensional Targeting," Phys. Rev. E 47, pp. 305-310.

Lachmann, G.V. (editor) (1961) Boundary Layer Control and Flow Control, vols. 1 and 2, Pergamon Press, Oxford. 
Lai, Y.-C., and Grebogi, C. (1993) "Synchronization of Chaotic Trajectories Using Control," Phys. Rev. E 47, pp. 2357-2360.

Lai, Y.-C., Deng, M., and Grebogi, C. (1993a) "Controlling Hamiltonian Chaos," Phys. Rev. E 47, pp. 86-92.

Lai, Y.-C., Grebogi, C., and Tél, T. (1994) "Controlling Transient Chaos in Dynamical Systems," in Towards the Harnessing of Chaos, ed. M. Yamaguchi, Elsevier, Amsterdam.

Lai, Y.-C., Tél, T., and Grebogi, C. (1993b) "Stabilizing Chaotic-Scattering Trajectories Using Control," Phys. Rev. E 48, pp. 709-717.

Liepmann, H.W., and Nosenchuck, D.M. (1982) "Active Control of Laminar-Turbulent Transition," J. Fluid Mech. 118, pp. 201-204.

Liepmann, H.W., Brown, G.L., and Nosenchuck, D.M. (1982) "Control of Laminar Instability Waves Using a New Technique," J. Fluid Mech. 118, pp. 187-200.

Lindner, J.F., and Ditto, W.L. (1995) "Removal, Suppression and Control of Chaos by Nonlinear Design," Appl. Mech. Rev. 48, pp. 795-808.

Löfdahl, L., Glavmo, M., Johansson, B., and Stemme, G. (1993) "A Silicon Transducer for the Determination of Wall-Pressure Fluctuations in Turbulent Boundary Layers," Appl. Scientific Res. 51, pp. 203-207.

Löfdahl, L., Kälvesten, E., and Stemme, G. (1994) "Small Silicon Based Pressure Transducers for Measurements in Turbulent Boundary Layers," Exp. Fluids 17, pp. 24-31. 
Lumley, J.L. (1991) "Control of the Wall Region of a Turbulent Boundary Layer," in Turbulence: Structure and Control, ed. J.M. McMichael, pp. 61-62, 1-3 April, Ohio State University, Columbus, Ohio.

Lüscher, E., and Hübler, A. (1989) "Resonant Stimulation of Complex Systems," Helv. Phys. Acta 62, pp. 544-551.

Mastrangelo, C. (1993) "Integration, Partition, and Reliability of Microelectromechanical Systems," invited oral presentation at the AIAA Third Flow Control Conference, Orlando, Florida, 6-9 July.

McMichael, J.M. (1993) "MEMS and Challenges of Flow Control," invited oral presentation at the AIAA Third Flow Control Conference, Orlando, Florida, 6-9 July.

McMichael, J.M. (1996) "Progress and Prospects for Active Flow Control Using Microfabricated Electromechanical Systems (MEMS)," AIAA Paper No. 96-0306, Washington, D.C.

Mehregany, M. (1993) "Overview of Microelectromechanical Systems," invited oral presentation at the AIAA Third Flow Control Conference, Orlando, Florida, 6-9 July.

Mehregany, M., DeAnna, R.G., and Reshotko, E. (1996) "Microelectromechanical Systems for Aerodynamics Applications," AIAA Paper No. 96-0421, Washington, D.C.

Moin, P. and Bewley, T. (1994) "Feedback Control of Turbulence," Appl. Mech. Rev. 47, pp. S3-S13. 
Nadolink, R.H., and Haigh, W.W. (1995) "Bibliography on Skin Friction Reduction with Polymers and other Boundary-Layer Additives," Appl. Mech. Rev. 48, pp. 351-459.

Narasimha, R., and Sreenivasan, K.R. (1979) "Relaminarization of Fluid Flows," in Advances in Applied Mechanics, vol. 19, ed. C.-S. Yih, pp. 221-309, Academic Press, New York.

Nelson, M.M., and Illingworth, W.T. (1991) A Practical Guide to Neural Nets, Addison-Wesley, Reading, Massachusetts.

Ott, E., Grebogi, C., and Yorke, J.A. (1990a) "Controlling Chaos ," Phys. Rev. Lett. 64, pp. 1196-1199.

Ott, E., Grebogi, C., and Yorke, J.A. (1990b) "Controlling Chaotic Dynamical Systems," in Chaos: Soviet-American Perspectives on Nonlinear Science, ed. D.K. Campbell, pp. 153-172, American Institute of Physics, New York.

Pomeau, Y., and Manneville, P. (1980) "Intermittent Transition to Turbulence in Dissipative Dynamical Systems," Commun. Math. Phys. 74, pp. 189-197.

Prandtl, L. (1904) "Über Flüssigkeitsbewegung bei sehr kleiner Reibung," Proc. Third Int. Math. Congr., pp. 484-491, Heidelberg, Germany.

Qin, F., Wolf, E.E., and Chang, H.-C. (1994) "Controlling Spatiotemporal Patterns on a Catalytic Wafer," Phys. Rev. Lett. 72, pp. 1459-1462.

Robinson, S.K. (1991) "Coherent Motions in the Turbulent Boundary Layer," Annu. Rev. Fluid Mech. 23, pp. 601-639. 
Romeiras, F.J., Grebogi, C., Ott, E., and Dayawansa, W.P. (1992) "Controlling Chaotic Dynamical Systems," Physica D 58, pp. 165-192.

Roos, F.W. (1996) ""Microblowing" for High-Angle-of-Attack Vortex Flow Control on a Fighter Aircraft," AIAA Paper No. 96-0543, Washington, D.C.

Schreck, S.J., Faller, W.E., and Luttges, M.W. (1995) "Neural Network Prediction of ThreeDimensional Unsteady Separated Flow Fields," J. Aircraft 32, pp. 178-185.

Sen, M., Wajerski, D., and Gad-el-Hak, M. (1996) "A Novel Pump for Low-Reynolds-Number Flows," J. Fluids Eng., to appear.

Sharatchandra, M.C., Sen, M., and Gad-el-Hak, M. (1996) "Navier-Stokes Simulations of a Novel Viscous Pump," J. Fluids Eng., to appear.

Shinbrot, T. (1993) "Chaos: Unpredictable Yet Controllable?" Nonlinear Science Today 3, pp. 1-8.

Shinbrot, T. (1995) "Progress in the Control of Chaos," Adv. Physics 44, pp. 73-111.

Shinbrot, T., and Ottino, J.M. (1993a) "Geometric Method to Create Coherent Structures in Chaotic Flows," Phys. Rev. Lett. 71, pp. 843-846.

Shinbrot, T., and Ottino, J.M. (1993b) "Using Horseshoes to Create Coherent Structures in Chaotic Fluid Flows," Bul. Am. Phys. Soc. 38, p. 2194. 
Shinbrot, T., Ditto, W., Grebogi, C., Ott, E., Spano, M., and Yorke, J.A. (1992a) "Using the Sensitive Dependence of Chaos (the "Butterfly Effect") to Direct Trajectories in an Experimental Chaotic System," Phys. Rev. Lett. 68, pp. 2863-2866.

Shinbrot, T., Grebogi, C., Ott, E., and Yorke, J.A. (1992b) "Using Chaos to Target Stationary States of Flows," Phys. Lett. A 169, pp. 349-354.

Shinbrot, T., Grebogi, C., Ott, E., and Yorke, J.A. (1993) "Using Small Perturbations to Control Chaos," Nature 363, pp. 411-417.

Shinbrot, T., Ott, E., Grebogi, C., and Yorke, J.A. (1990) "Using Chaos to Direct Trajectories to Targets," Phys. Rev. Lett. 65, pp. 3215-3218.

Shinbrot, T., Ott, E., Grebogi, C., and Yorke, J.A. (1992c) "Using Chaos to Direct Orbits to Targets in Systems Describable by a One-Dimensional Map," Phys. Rev. A 45, pp. 4165-4168.

Singer, J., Wang, Y.-Z., and Bau, H.H. (1991) "Controlling a Chaotic System," Phys. Rev. Lett. 66, pp. 1123-1125.

Sirovich, L., and Deane, A.E. (1991) "A Computational Study of Rayleigh-Bénard Convection. Part 2. Dimension Considerations," J. Fluid Mech. 222, pp. 251-265.

Tai, Y.-C. (1993a) "Silicon Micromachining and Micromechanics," invited oral presentation at the AIAA Third Flow Control Conference, Orlando, Florida, 6-9 July.

Tang, J., and Bau, H.H. (1993a) "Stabilization of the No-Motion State in Rayleigh-Bénard Convection through the Use of Feedback Control," Phys. Rev. Lett. 70, pp. 1795-1798. 
Tang, J., and Bau, H.H. (1993b) "Feedback Control Stabilization of the No-Motion State of a Fluid Confined in a Horizontal Porous Layer Heated from Below," J. Fluid Mech. 257, pp. $485-505$.

Thibert, J.J., Reneaux, J, and Schmitt, V. (1990) "ONERA Activities on Drag Reduction," Proceedings of the Seventeenth Congress of the International Council of the Aeronautical Sciences, vol. 1, pp. 1053-1064, Paper No. ICAS-90-3.6.1, American Institute of Aeronautics and Astronautics, Washington, D.C.

Vargo, S.E., and Muntz, E.P. (1996) "A Simple Micromechanical Compressor and Vacuum Pump for Flow Control and Other Distributed Applications," AIAA Paper No. 96-0310, Washington, D.C.

Viswanath, P.R. (1995) "Flow Management Techniques for Base and Afterbody Drag Reduction," Prog. Aero. Sci. 32, pp. 79-129.

Wagner, R.D., Fischer, M.C., Collier, F.S., Jr., and Pfenninger,W. (1990) "Supersonic Laminar Flow Control on Commercial Transports," Proceedings of the Seventeenth Congress of the International Council of the Aeronautical Sciences, vol. 1, pp. 1073-1089, Paper No. ICAS-903.6.3, American Institute of Aeronautics and Astronautics, Washington, D.C.

Wang, Y., Singer, J., and Bau, H.H. (1992) "Controlling Chaos in a Thermal Convection Loop," J. Fluid Mech. 237, pp. 479-498.

Wells, C.S. (1969) Viscous Drag Reduction, Plenum Press, New York. 
Wiltse, J.M., and Glezer, A. (1993) "Manipulation of Free Shear Flows Using Piezoelectric Actuators," J. Fluid Mech. 249, pp. 261-285.

Wilkinson, S.P. (1990) "Interactive Wall Turbulence Control," in Viscous Drag Reduction in Boundary Layers, eds. D.M. Bushnell and J.N. Hefner, Progress in Astronautics and Aeronautics, vol. 123, pp. 479-509, AIAA, Washington, D.C.

Williams, D.R., and Amato, C.W. (1989) "Unsteady Pulsing of Cylinder Wakes," in Frontiers in Experimental Fluid Mechanics, Lecture Notes in Engineering, vol. 46, pp. 337-364, SpringerVerlag, Berlin.

Yun, W. (1993) "System Considerations for Integration of Microsensors and Electronics," invited oral presentation at the AIAA Third Flow Control Conference, Orlando, Florida, 6-9 July. 


\section{FIGURE CAPTIONS}

Figure 1. Classification of Flow Control Strategies.

Figure 2. Different Control Loops for Active Flow Control.
(a) Predetermined, Open Loop.
(b) Reactive, Feedforward, Open Loop.
(c) Reactive, Feedback, Closed Loop.

Figure 3. The OGY Method for Controlling Chaos.

Figure 4. Elements of a Neural Network. 


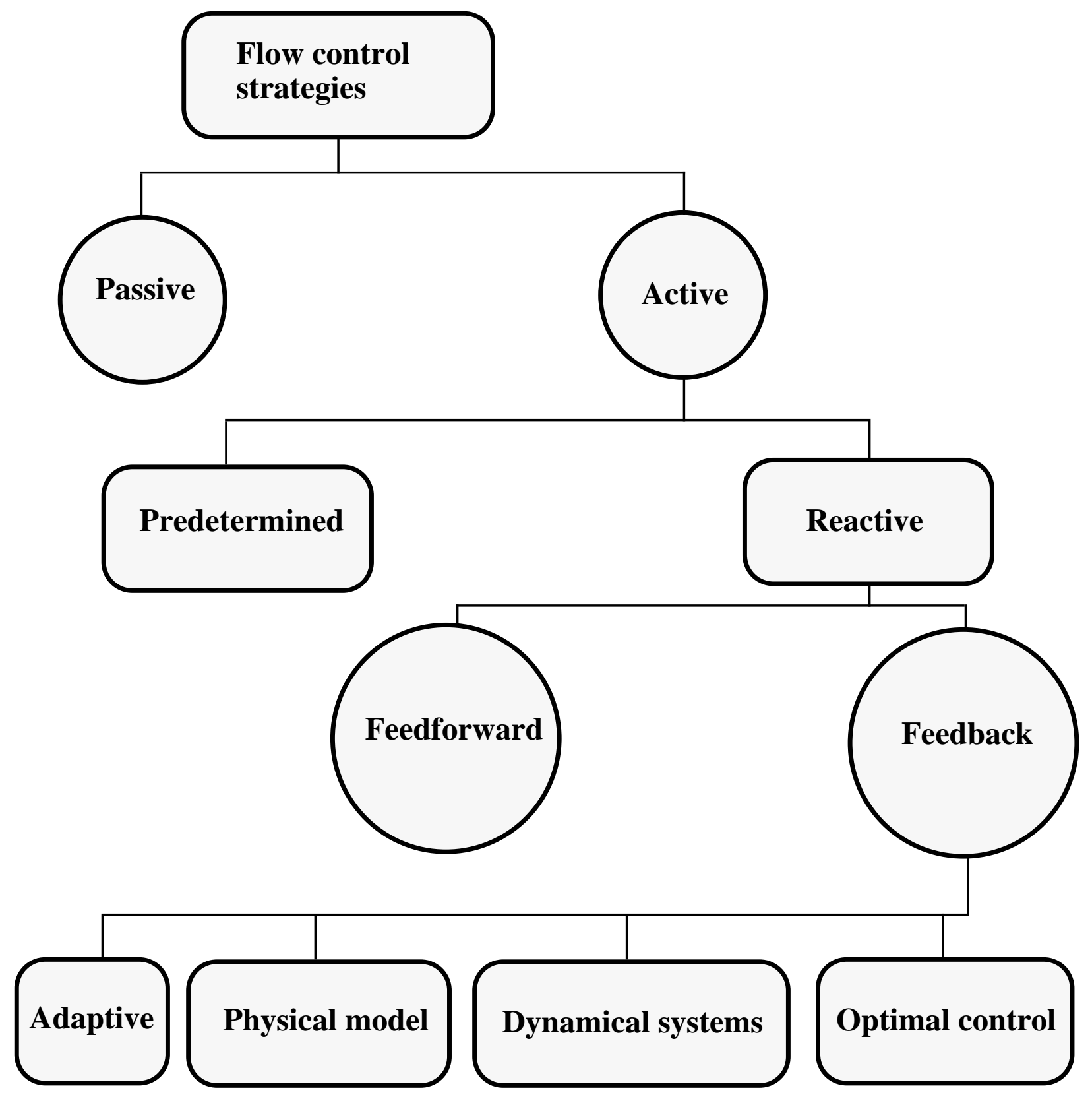

Figure 1. Classification of Flow Control Strategies. 

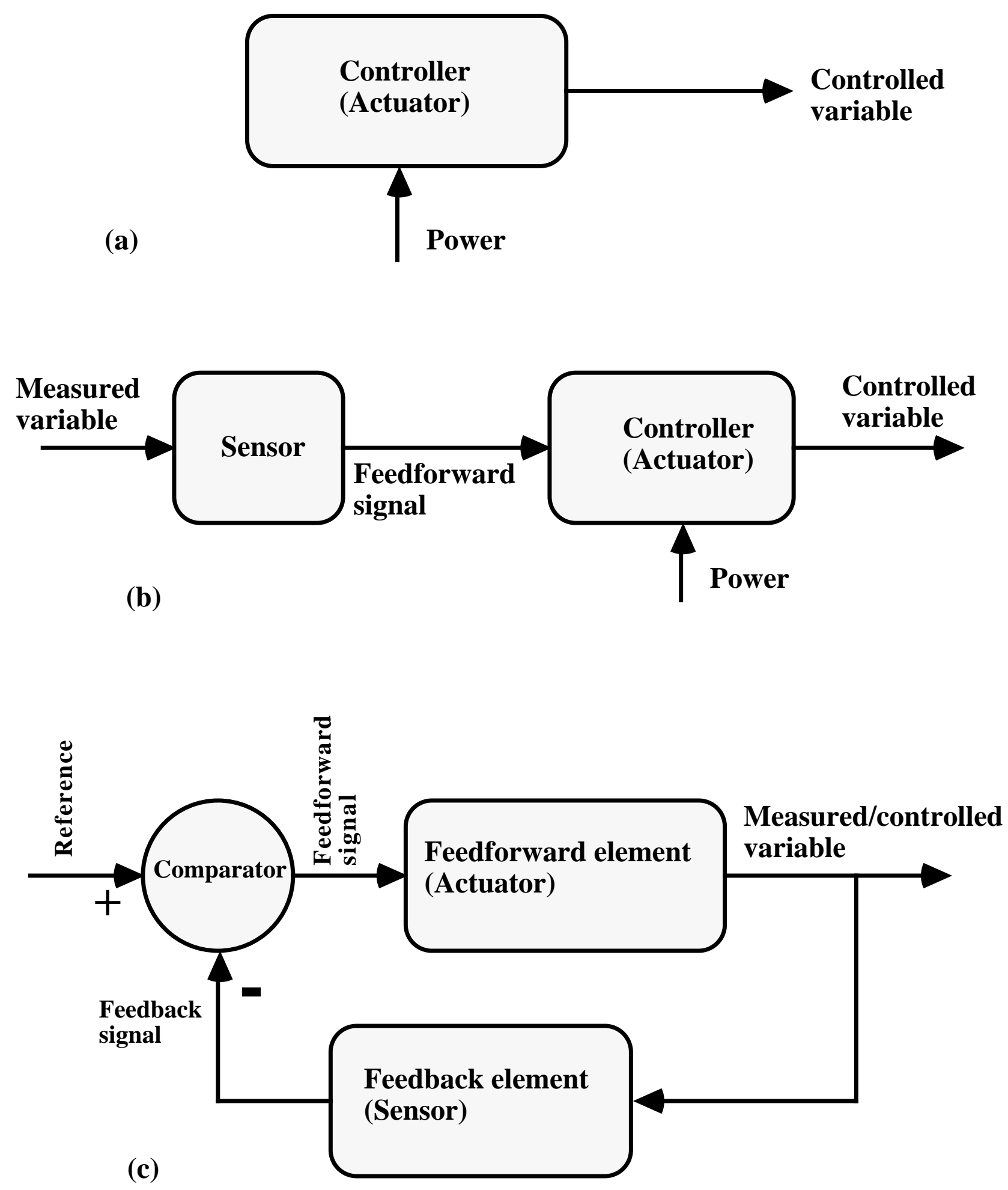

Figure 2. Different Control Loops for Active Flow Control. 


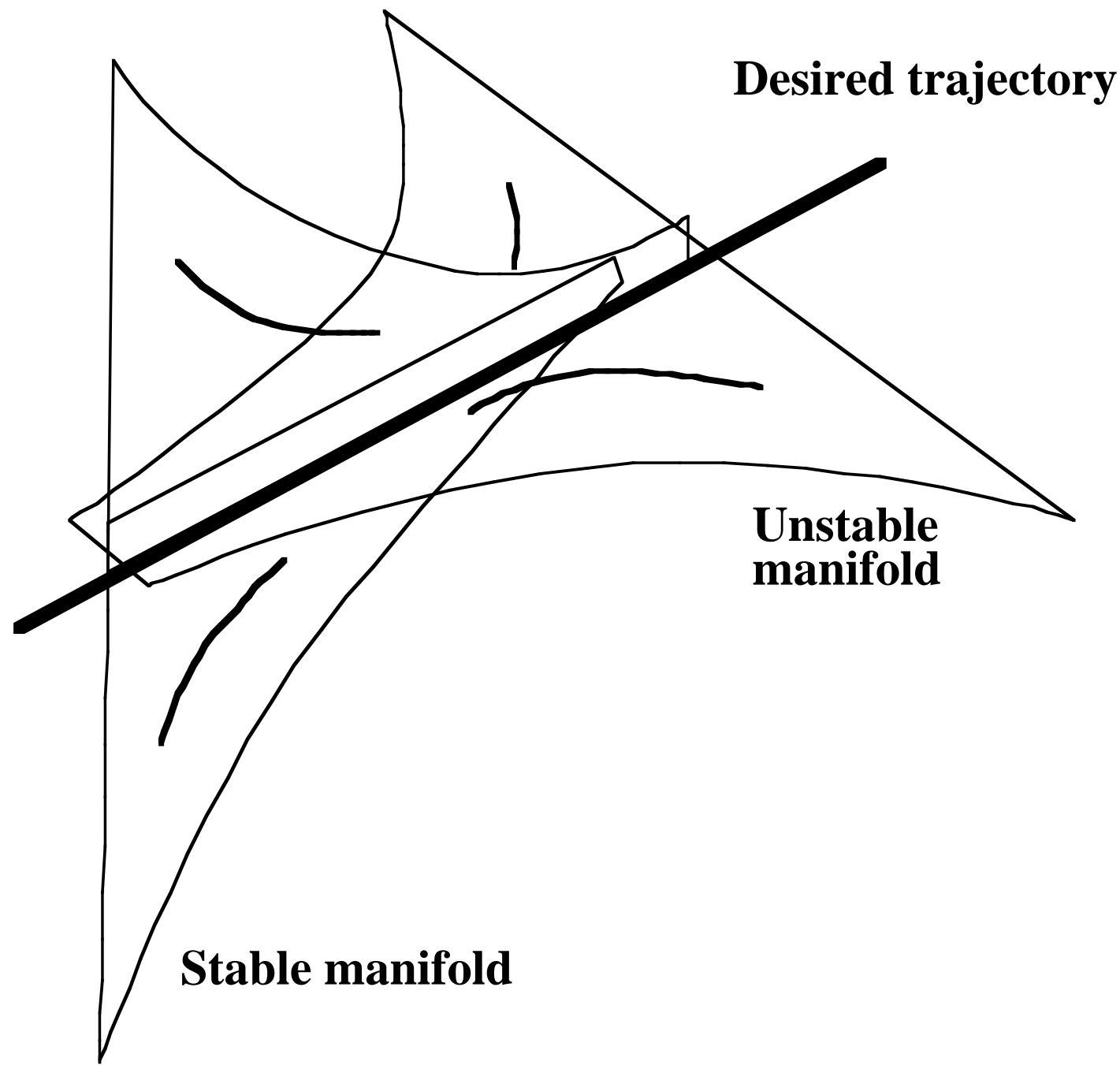

Figure 3. The OGY Method for Controlling Chaos. 


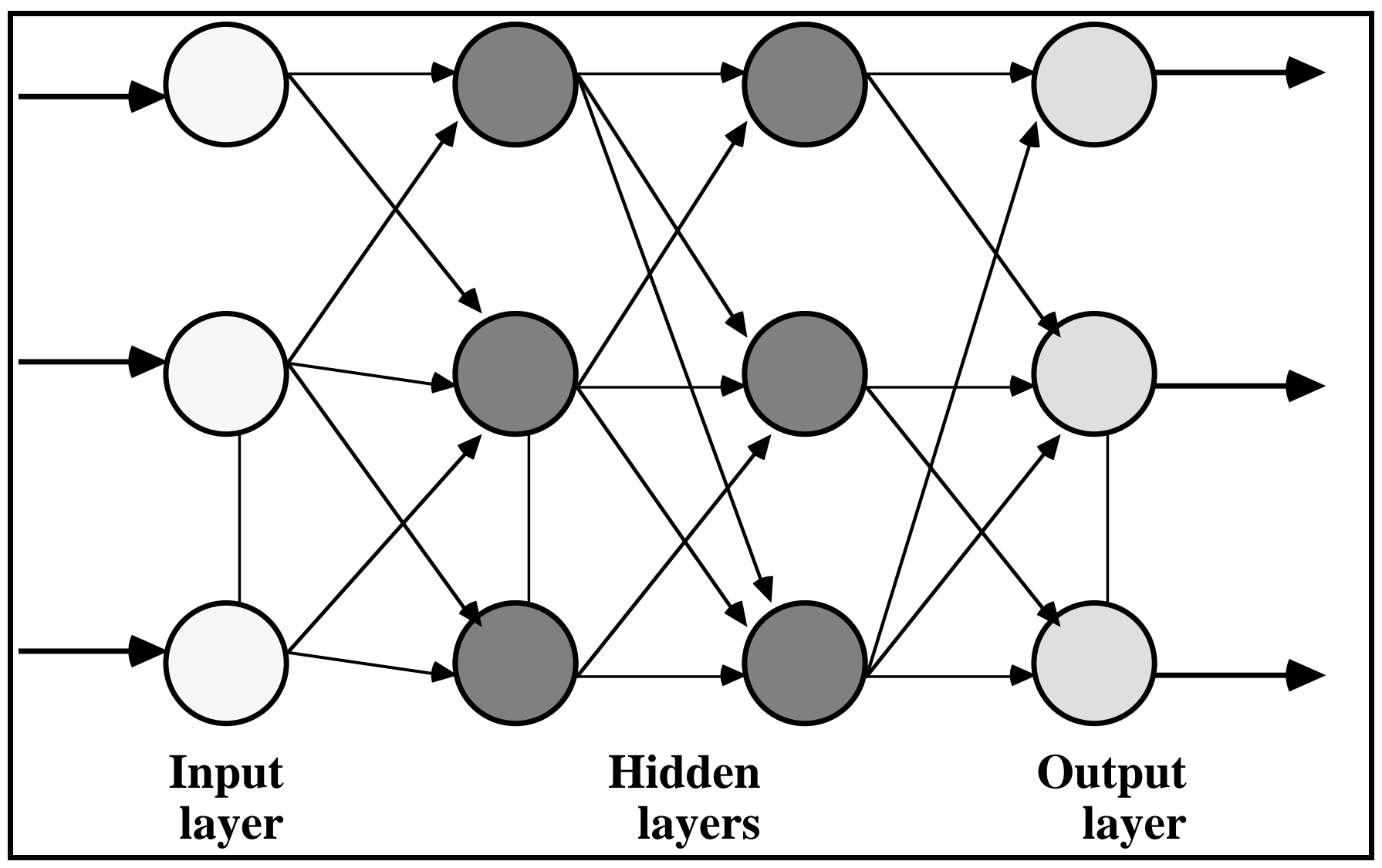

Figure 4. Elements of a Neural Network. 